\title{
THE FORTY-FIFTH REGULAR MEETING OF THE SAN FRANCISCO SECTION
}

The forty-fifth regular meeting of the San Francisco Section of this Society was held at Stanford University on April 4, 1925. Professor E. R. Hedrick presided at the morning session and Professor A. F. Carpenter at the afternoon session. The total attendance was thirty-six, including the following twenty-six members of the Society:

Allardice, A. D. B. Andrews, Bernstein, Blichfeldt, Buck, Cajori, A. F. Carpenter, Daus, M. W. Haskell, E. R. Hedrick, Hoskins, Hotelling, Glenn James, Lehmer, Sophia Levy, E. W. Mc Donald, J. H. Mc Donald, W.A.Manning, Mathewson, Moreno, F.R.Morris, Noble, Pauline Sperry, Stager, A. R.Williams, Wong.

It was voted that, beginning with the next Fall meeting. the retiring Chairman deliver an address before the Section.

The dates and places of the next three regular meetings of the Section were fixed as follows: June 19, 1925, University of Oregon; October 31, 1925, University of California; April 3, 1926, Stanford University.

Titles and abstracts of papers read at this meeting follow. The papers of Dr. Barter, Dr. Mullemeister, and of Professors Bateman, Bell, Moritz were read by title.

1. Dr. John Barter: Notes on algebraic theory. Part I. The linear operator.

The present paper developes the theory of linear transformations in such directions as seem essential to application to the general algebraic theory. The theory of $\lambda$-matrices is treated with some detail from a new angle and by a method applicable to $\lambda$-matrices of any degree. The new conception of poles of different orders is made a basis for a classification of these matrices. The classic theory of elementary divisors appears as a particular case of the general theory. Automorphic transformations are treated incidentally and in an introductory manner from this standpoint. 
2. Professor Harry Bateman: An extension of Lagrange's expansion.

When $z$ is defined by means of the equation $z=a+x \Phi(z)$, Lagrange's theorem furnishes the expansion, in ascending powers of $x$, of an arbitrary function $f(z)$. The present extension of the expansion gives a corresponding series for the definite integral

$$
\int_{a}^{z} F[a+x \Phi(\boldsymbol{\tau})-\boldsymbol{\tau}, \boldsymbol{\tau}] d \boldsymbol{\tau}
$$

where $F$ is an arbitrary function of its two arguments. The series was suggested by a generalization of $\mathrm{E}$. T. Whittaker's solution of Laplace's equation.

3. Professor E. T. Bell: The chi functions of Glaisher and class numbers.

The function $\chi_{r}(n)$ of Glaisher is the sum of the $r$ th powers of all Gaussian primary complex numbers having norm $n$. This function is here expressed in terms of binary quadratic class numbers, and several new class number relations are indicated.

4. Professor B. A. Bernstein: On the existence of fields in boolean algebras.

The author proves that $x y^{\prime}+x^{\prime} y, x y ; x y+x^{\prime} y^{\prime}, x+y$ ( $x^{\prime}$ the negative of $x$ ) are the only pairs of operations for which the elements of a two-element boolean algebra are a field, and that there exist no field operations expressible in terms of addition, multiplication, and negation for the totality of the elements of any other boolean algebra.

5. Professor H. F. Blichfeldt: On the minimum value of positive real quadratic forms in 6 variables.

Let $f$ represent a positive real quadratic form in $n$ variables, having a determinant $D$. Then we may substitute integers, not all zero, for the variables such that the numerical value of $f$ is not greater than $\gamma_{n} \cdot \sqrt[n]{|D|}$; where $\gamma_{n}$ is a function of $n$ only. Superior limits to $\gamma_{n}$ have been given for any $n$, as well as the exact minima for $n=2,3,4,5$ (cf. Transactions of this society, vol. 15 (1914), p. 233). In the present paper the author shows that the exact. minimum for $n=6$ is $2 / \sqrt[6]{3}$. 
6. Professor Florian Cajori: American contributions to mathematical notations.

The author makes reference to the Maya numeral notation marking the earliest regular use of a sign for zero and the principle of local value, the Peruvian knot records, the dollar mark, a sporadic notation for radicals, the sign $=$ for equivalence, B. Peirce's symbols for $\pi$, $e$ and $i$, C. S. Peirce's notation in symbolic logic, the $\doteq$ for approach to the limit, J. W. Gibbs's vector notation, E. H. Moore's notation in general analysis. As yet, none of these have found permanent adoption in Europe, except perhaps the dollar mark.

7. Professor Florian Cajori: Early appearance in print of the dollar mark.

The author shows that the dollar mark originated from the superposition of the letters $p$ and $s$ in "pesos". The frequent statement that the first printed appearance of the sign $\$$ to represent dollars is in Chauncey Lee's American Accomptant, 1797, was shown to be inaccurate. The \$ occurred in print in the Scholars' Arithmetic of Daniel Adams (4th. ed., 1807) and began to be used freely in newspapers about 1810 .

8. Professor A.F. Carpenter: Two point-line correspondences determined by a ruled surface.

By means of the two quadrics which osculate the two sheets of the flecnode surface of a ruled surface along a pair of corresponding line elements, two point-line correspondences of space are set up. These correspondences are such that all points of space are made to correspond, in two ways, to the lines of a congruence.

The paper also determines for numerous point loci of space, the corresponding line loci.

9. Professor P. H. Daus: Certain recursion formulas connected with the solution of $x^{2}+y^{2}=z^{2}$.

If

$$
s_{1}=\left(x_{1}, y_{1}, z_{1}\right)
$$

is the smallest primitive solution of

$$
x^{2}+y^{2}=z^{2},
$$


where

$$
y=x+m,
$$

then all solutions of these equations when $m$ is a prime or the power of a prime are given by the formulas

$$
\begin{aligned}
& s_{2 n+1}=p s_{2 n}-p s_{2 n-1}+s_{2 n-2} ; \\
& s_{2 n+2}=q s_{2 n+1}-q s_{2 n}+s_{2 n-1}
\end{aligned}
$$

where $p$ and $q$ depend on $s_{1}$. The solutions divide themselves into two sets, according as $n$ of $s_{n}$ is even or odd. Within each set the solutions are connected by the relation

$$
s_{n+2}=7 s_{n+1}-7 s_{n}+s_{n-1},
$$

independent of the value of $m$.

10. Professor M. W. Haskell: Autopolar configurations in space.

The method of finding multiply self-polar curves presented to the International Congress at Toronto can be extended to configurations in space. There are certain modifications to be noted, in particular with regard to the nature of the combination of a polarity with a collineation group of which the quadric surface in question is an invariant. Perspective reflexions always give rise to new polarities, while skew reflexions are of two kinds. If the invariant lines of a skew reflexion are polar to each other, then the product of the collineation and the given polarity is a second polarity; while if they are self-polar (i. e. tangent to the given quadric surface) the resulting correlation is a null-system.

For example, the Kummer surface is invariant under a group of 16 collineations of period 2 (all skew reflexions) and 16 correlations, of which 10 are polarities with respect to quadric surfaces and 6 are polarities with respect to nullsystems.

11. Professor E. R. Hedrick: Extensions of Picard's theorem to non-analytic functions.

The author points out that there are a number of rather general cases in which extensions of Picard's theorem to non-analytic functions are possible, and several specific forms of the extended theorem are given. Some limitations on possible extensions are stated, for functions that are non-analytic. 
12. Dr. Harold Hotelling: Theory of mine economics.

The equations of competition are modified in the case of exhaustible resources by the addition to each equation of a term of the form $\lambda_{i} e^{\delta t}$, where $\delta$ is the force of interest and the $\lambda_{i}$ 's depend upon the amounts of the resource in the hands of the various entrepreneurs. Although the the equations of competition of inexhaustible resources admit of solution when demand is assumed perfectly inelastic, this assumption in the present case leads to an absurdity. Though natural resources may in some cases be exploited too rapidly under competition, the analysis shows that in other cases the exploitation is slower than the public interest requires.

13. Dr. Harold Hotelling: Manifolds of states of motion as multiple-sheeted spaces.

The boundaries of a pair of plane rings which link form the branch curves of a $(2 p+2)$-sheeted space homeomorphic with the manifold of states of motion on an orientable surface of genus $p>0$. For a one-sided surface of the same connectivity the representation is by branch curves of the same type in a space of $2 p+4$ sheets. For the representation of the states of motion on a one-sided surface of the first kind the branch system consists of these four curves and two others, each of the latter linking doubly the other and also one of the plane rings.

14. Professor Glenn James: On the solution of equations of degree higher than the fifth.

This paper developes a formula for one root of the equation

$$
x^{n}+\alpha_{1} x^{n-1}+\alpha_{2} x^{n-2}+\cdots+\alpha_{n}=0,
$$

when $n$ is odd or when $n$ is even and $\alpha_{n}$ is negative, and presents a theoretically possible method of solving this equation for any positive integral value of $n$.

15. Professor D. N. Lehmer: A new method of finding factors of numbers.

The author's method is an improvement on the well known method which depends on the theory of quadratic residues. By means of a set of stencils the divisors 
[October,

which belong to a set of linear forms are made apparent at once. The method is available for numbers as high as ten billions.

16. Professor J. H. McDonald: The equation of the elliptic cylinder.

The solution of the linear differential equation of the second order which belongs to the elliptic cylinder is given for the general case. The equation is of a type not derivable by specialization from the hypergeometric differential equation and has a non-regular singular point at infinity. The problem is to determine the characteristic exponent and the coefficients of the series giving the solution, the coefficients being functions of the characteristic exponent.

The differential equation depends on two parameters and is first transformed into a Riccati equation which necessarily has a periodic solution. This is obtained in the form of a series of powers of one of the parameters, the coefficients being periodic functions of the independent variable. The sum of the constant terms in these coefficients is a power series in one parameter, the coefficients being rational functions of the other. This series is a branch of the characteristic exponent. The coefficients of the solution are next given explicitly by means of infinite determinants. The equation for the characteristic exponent which arises in determining the coefficients is put in a form such that all branches of the exponent can be determined.

17. Professor J. H. McDonald: The periodic solutions of the equation of the elliptic cylinder.

The solution of the equation of the elliptic cylinder is not periodic in general but only if a special relation holds between the parameters. The forms of a few of the periodic solutions have been determined but by a method which does not seem capable of being extended to the general case. In this paper a method is given by which they may all be obtained, and the equation between the parameters which must be satisfied for the existence of the periodic solution is completely solved. The periodic solutions are known to be the solutions of a particular integral equation. Its general solution and the characteristic numbers are accordingly determined. 
18. Professor J.H. McDonald: On the periodic solution of a certain differential equation.

A problem in motion under a periodic disturbing force leads to the differential equation

$$
\frac{d^{2} x}{d t^{2}}-m x\left(\frac{d x}{d t}\right)^{2}+n^{2} x=a \sin p t .
$$

This equation has a single periodic solution if $n^{2} / p^{2}$ is not equal to the square of an odd integer. The solution is of the form

$$
\sum_{1}^{\infty} \alpha_{k} \sin (2 k-1) p t
$$

and the coefficients $a_{k}$ are all determined. The method used is that of solving a succession of differential equations which have the given equation as a limiting form; the solution at each step being chosen so that a succession of functions is determined which converges to a solution of the problem.

19. Professor J. H. McDonald: The transformation of elliptic integrals.

The theory of transformation of elliptic integrals given previously by the writer for the general case is applied to the transformation of the seventh order. The modular equation is obtained in the appropriate form and the results compared with those of Kiepert and Joubert.

20. Professor J. H. McDonald: On the reduction of the singularities of algebraic curves.

Halphen enunciated the theorem that an algebraic curve can be transformed birationally into one whose multiple points are double points with distinct tangents. His proof was defective but proofs have been given by Poincaré, Bertini, and others. A short proof of the theorem is given by determining a space curve whose projection is the required curve with reduced singularities.

21. Professor R. E. Moritz: On the kinematic construction of higher plane curves.

Professor Moritz considers the various loci which result from a composition of certain elementary motions which 
can readily be effected kinematically. The equations of these motions give in each case the simplest possible parametric equations of the loci under consideration. This paper will appear in the American Mathematical Monthly.

22. Dr. Hermance Mullemeister: A projection of the lines in space into pairs of lines in a plane.

The four-dimensional line-space is projected from two centers $C_{1}$ and $C_{2}$ into pairs of lines in a plane. An axial complex on $C_{1} C_{2}$ is projected into a pencil of lines on $C_{0}$, the intersection of $C_{1} C_{2}$ and the picture plane $\alpha$. A flat pencil is projected into two perspective pencils in $\alpha$, whose vertices $P_{1}$ and $P_{2}$ are collinear with $C_{0}^{\prime}$ and whose axis of perspectivity is the intersection of the two planes. Two pencils $P_{1}$ and $P_{2}$ in $\alpha$ which are projective, but not perspective, constitute the image of a quadratic ruled system, whose axes are $P_{1} C_{1}$ and $P_{2} C_{2}$. A quadratic ruled system which does not pass through $C_{1}$ and $C_{2}$, is projected into two projective line-conics which have two common tangents through $C_{0}$.

A line-field in a plane $\beta$ is projected into two perspective line-fields in $\alpha$ having $C_{0}$ as center of perspectivity and the intersection of $\alpha$ and $\beta$ as axis of perspectivity. A line-sheaf on $O$ is projected into two pencils whose vertices are the projections of $O$ from $C_{1}$ and $C_{2}$, which are collinear with $C_{0}$. A bilinear congruence is projected into pairs of lines such that to every linepencil $\left(l_{1}\right)$ corresponds a line-conic $\mu_{2}^{2}$, and to every linepencil $\left(l_{2}\right)$ corresponds a line-conic $\mu_{1}^{2}$.

B. A. Bernstein, Secretary of the Section. 